assessed in line with the RA MRI scoring system (summed as RAMRIS-inflammation). Both IMB and RAMRIS-inflammation were dichotomised into positive/negative using data from age-matched symptom-free controls as a reference. Cox regression analysed the association of IMB with progression to clinical arthritis; multivariable analyses were used to adjust for RAMRIS-inflammation which is known to associate with progression to clinical arthritis. Analyses were repeated stratified for ACPA-status, since ACPA-positive and ACPA-negative RA are considered separate entities with differences in pathophysiology.

Results: The baseline MRIs showed $\geq 1 \mathrm{IMB}$ in $35 \%$ of CSA-patients. Patients with IMB were more likely to also have synovitis (OR $2.5(95 \% \mathrm{Cl} 1.2-4.9)$ ) and tenosynovitis (8.9 (3.4-22.9)) on forefoot MRI, but not osteitis $(0.9$ (0.5-1.8)). Patients were followed for median 25 months (IQR 19-27). IMB-positive patients developed clinical arthritis more often than IMB-negative patients (HR 3.0 (1.94.8)). This association was independent of RAMRIS-inflammation (adjusted HR 2.2 (1.4-3.6)). In stratified analyses, IMB was more frequent in ACPA-positive than in ACPA-negative CSA (68\% vs. $30 \%$, p<0.001). Moreover IMB predicted clinical arthritis development in ACPA-positive CSA (HR 2.5 (1.1-5.7)) but not in ACPA-negative CSA patients (1.0 (0.5-2.2)).

Conclusion: One-third of CSA patients have IMB. IMB is frequently present in conjunction with subclinical synovitis and tenosynovitis. It precedes the development of clinical arthritis, and in particular the development of ACPA-positive RA. These results reinforce the notion that not only intra- but also juxta-articular synovial inflammation is involved in the development of RA

Disclosure of Interests: None declared

DOI: 10.1136/annrheumdis-2021-eular.1710

\section{EVOLUTION OF ANTI-MODIFIED PROTEIN ANTIBODY RESPONSES CAN BE DRIVEN BY CONSECUTIVE EXPOSURE TO DIFFERENT POST-TRANSLATIONAL MODIFICATIONS}

M. Volkov ${ }^{1}$, A. S. B. Kampstra ${ }^{1}$, K. van Schie ${ }^{1}$, A. Kawakami ${ }^{2}$, M. Tamai ${ }^{2}$, S. Y. Kawashiri ${ }^{3}$, T. Maeda ${ }^{4}$, T. Huizinga ${ }^{1}$, R. Toes ${ }^{1}$, D. van der Woude ${ }^{1}{ }^{1}{ }^{1}$ Leiden University Medical Center, Rheumatology, Leiden, Netherlands; ${ }^{2}$ Nagasaki University Graduate School of Biomedical Sciences, Immunology and Rheumatology, Nagasaki, Japan; ${ }^{3}$ Nagasaki University Graduate School of Biomedical Sciences, Community Medicine, Nagasaki, Japan; ${ }^{4}$ Nagasaki University Graduate School of Biomedical Sciences, General Medicine, Nagasaki, Japan

Background: Besides anti-citrullinated protein antibodies (ACPA), rheumatoid arthritis patients (RA) often display autoantibody reactivities against other post-translationally modified (PTM) proteins, more specifically carbamylated and acetylated proteins. Immunizing mice with one PTM results in an anti-modified protein antibody (AMPA) response recognizing multiple PTMs. Furthermore, human AMPA, isolated based on their reactivity to one PTM, cross-react with other PTMs at the monoclonal and polyclonal level. However, it is unclear whether the AMPA reactivity profile is "fixed" in time, or whether consecutive exposure to different PTMs can shape the evolving AMPA-response.

Objectives: To investigate the evolution of the AMPA response in mice with controlled exposure to PTMs as well as in AMPA-positive humans.

Methods: Mice were immunized with acetylated (or carbamylated) protein (ovalbumin) twice or cross-immunized with an acetylated and then a carbamylated protein (or vice versa) and their serum was analyzed for AMPA responses with ELISA using a different backbone protein (fibrinogen) bearing the same modifications. Longitudinally collected serum samples of human individuals at risk of RA and with early RA were tested to investigate the evolution of the AMPA responses in humans.

Results: Mice immunized twice with either solely acetylated or solely carbamylated ovalbumin (AcOVA or CaOVA) developed reactivity against both acetylated and carbamylated antigens. Irrespective of the PTM used for the first immunization, a booster immunization with the other PTM resulted in increased titers to the second/booster PTM (Figure 1A), suggesting that immunization with a defined PTM-antigen leads to the generation of anti-PTM memory B cells able to cross-recognize other PTMs. Furthermore, immunizing with CaOVA and boosting with AcOVA (or vice versa) skewed the overall AMPA-response profile towards a relatively higher reactivity against the "booster" PTM (Figure 1B). Human data also illustrated dynamic changes in AMPA reactivity profiles in both individuals at risk of RA and in early RA patients (not shown).

Conclusion: The relationship between different reactivities within the AMPA response is dynamic. The initial exposure to a PTM antigen induces cross-reactive response that can be boosted by the same or other PTMs. The overall reactivity pattern can be skewed by subsequent exposure to other PTMs. These data might explain temporal changes in the reactivity profile of the AMPA response and point to the possibility that the PTM responsible for the initiation of the AMPA response may differ from the PTM predominantly recognized later in time.
Cross-boosting and skewing of AMPA responses in immunized mice
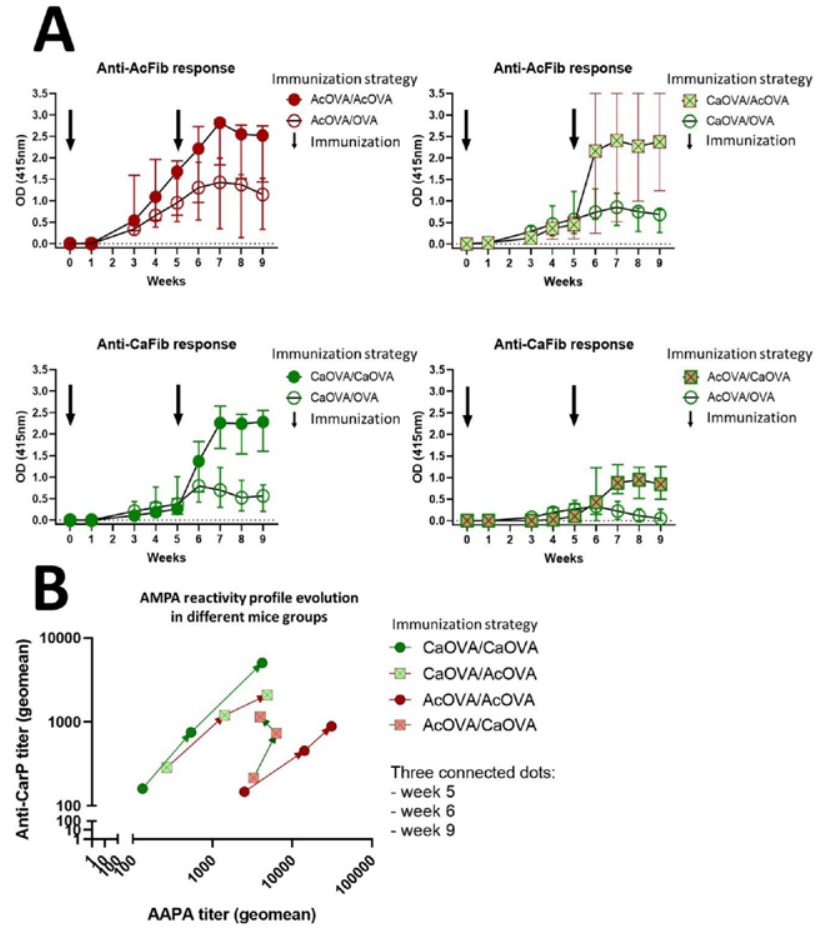

Disclosure of Interests: None declared DOI: 10.1136/annrheumdis-2021-eular.1743

\section{POS0387 ACPA STATUS CORRELATES WITH DIFFERENTIAL IMMUNE PROFILE OF RHEUMATOID ARTHRITIS PATIENTS}

A. Floudas ${ }^{1}$, M. Canavan ${ }^{1}$, T. McGarry' ${ }^{1}$, V. Krishna ${ }^{2}$, S. Nagpal ${ }^{2}$, D. Veale ${ }^{3}$, U. Fearon ${ }^{1}{ }^{1}$ Trinity Biomedical Sciences Institute, Molecular Rheumatology, DUBLIN, IRELAND, Ireland; ${ }^{2}$ Janssen Research \& Development, Immunology, Spring House, United States of America; ${ }^{3}$ St Vincent's University Hospital, UCD, EULAR Centre of Excellence, Centre for Arthritis and Rheumatic Diseases, Dublin, Ireland

Background: Rheumatoid arthritis (RA) is a progressive erosive autoimmune disease that affects $1 \%$ of the world population. Anti-citrullinated protein autoantibodies (ACPA) are routinely used for the diagnosis of RA, however $20-30 \%$ of patients are ACPA negative. ACPA status is a delineator of RA disease endotypes with similar clinical manifestation but potentially different pathophysiology. Elucidating the underlying mechanisms of disease pathogenesis could inform a treat to target approach for both ACPA-positive and ACPA-negative RA patients. Objectives: To identify peripheral blood and synovial tissue immune population differences that associate with RA disease endotype.

To identify unique RA patient synovial tissue gene signatures and enriched pathways that correlate with ACPA status.

Methods: Detailed high dimensionality flow cytometric analysis with supervised and unsupervised algorithm analysis of ACPApos and ACPAneg RA patient peripheral blood and synovial tissue single cell suspensions. Ex vivo peripheral blood and synovial tissue $T$ cell stimulation and cytokine production characterisation. RNAseq analysis with specific pathway enrichment analysis of APCApos and ACPAneg RA patient synovial tissue biopsies.

Results: Detailed profiling based on high dimensionality flow cytometric analysis of key peripheral blood and synovial tissue immune populations including B cells, T follicular helper (Tfh) cells, T peripheral helper cells (Tph) and CD4 $T$ cell proinflammatory cytokine responses with supervised and unsupervised algorithm analysis revealed unique RA patient peripheral blood $B$ cell and Tfh cell profiles. ACPApos RA patients were characterised by significantly $\left({ }^{*} \mathrm{P}=0.03\right)$ increased frequency of $\mathrm{Tfh}\left(\mathrm{CXCR} 5^{+} \mathrm{CD} 4^{+}\right)$cells and distinct clustering influenced by increased switched $\left(\operatorname{Ig} D^{-} C D 27^{+}\right)$and DN (IgD $\left.C D 27^{-}\right)$ memory B cells compared to APCAneg RA patients. Surprisingly synovial tissue B cell subpopulation distribution was similar between ACPAneg and ACPApos RA patients, with significant accumulation of switched and double negative memory B cells, highlighting a key role for specific B cell subsets in both disease endotypes. Interestingly, synovial tissue CD4 T cell 